\title{
CPED: A Chinese Positive Emotion Database for Emotion Elicitation and Analysis
}

\author{
Yulin Zhang, Guozhen Zhao, Yan Ge, Yezhi Shu, Dan Zhang, Yong-Jin Liu, and Xianghong Sun
}

\begin{abstract}
Positive emotions are of great significance to people's daily life, such as human-computer/robot interaction. However, the structure of extensive positive emotions is not clear yet and effective standardized inducing materials containing as many positive emotional categories as possible are lacking. Thus, this paper aims to establish a Chinese positive emotion database (CPED) to (1) effectively elicit positive emotion categories as many as possible, (2) provide both the subjective feelings of different positive emotions and a corresponding peripheral physiological database, and (3) explore the structure and framework of positive emotion categories. 42 video clips of 16 positive emotion categories were screened from more than 1000 online clips. Then a total of 312 participants watched and rated these video clips during which PPG and GSR signals were recorded. 34 video clips that met hit rate and intensity standards were systemically clustered into four emotion categories (empathy, fun, creativity and esteem). Eventually, 22 film clips of these four major categories formed the CPED database. 113 features from PPG and GSR signals were extracted and entered into a SVM classifier that serves as a baseline classification method. A classification accuracy of $45.84 \%$ for four major categories of positive emotions was achieved.
\end{abstract}

Index Terms-Positive emotion, emotional film clip, emotion elicitation, PPG, GSR

\section{INTRODUCTION}

$\mathrm{T}^{\mathrm{r}}$ raditional emotion categories put emphasis on negative emotion types (mainly used for psychological disease diagnosis and analysis) and usually treat positive emotion as a homogenous group, e.g., in Ekman's six basic emotions (happiness, sadness, fear, anger, surprise and disgust) [1], only happiness is generally recognized as positive emotion. However, with the development of internet social activities and human computer/robot interaction, positive emotions play more and more important roles in people's daily life [2] and have raised great concern with the development of positive psychology [3]. Positive emotions have positive effects. According to the "broaden-andbuilt" theory of positive emotions [4], the "broaden" function of positive emotions is to expand individual's immediate thinking and action scope including attention, cognition and problem solving. The "built" function of positive emotions is to construct individual's sustainable resources including psychological and social resources [5]. For example, it was reported that participants under positive emotional arousal showed continuous increase in memory for peripheral details [6]. Moreover, the experience of positive emotions could improve the perceived social connections [7]. Therefore, exploring the mechanism, recognizing accurately and applying positive emotions are of vital importance, since positive emotions have been proven to be a key factor in predicting life satisfaction and well-being [8].

- Y. Zhang, G. Zhao, Y. Ge and X. Sun are with the CAS Key Laboratory of Behavioral Science, Institute of Psychology, Beijing 100101, P. R. China, and with University of Chinese Academy of Sciences, Beijing 100049, P. R. China (E-mail: zhangyulin@psych.ac.cn,zhaogz@psych.ac.cn, gey@psych.ac.cn, sunxh@psych.ac.cn).

- Y.Shu and Y-J. Liu are with Beijing National Research Center for Information Science and Technology, Department of Computer Science and Technology, Tsinghua University, Beijing 100084, P. R. China. (E-mail: shuyz19@mails.tsinghua.edu.cn, liuyongjin@tsinghua.edu.cn).

- D. Zhang is with the Department of Psychology, Tsinghua University, Beijing 100084, P. R. China. (E-mail:dzhang@tsinghua.edu.cn).

- G. Zhao is the corresponding author.
Positive emotions have been defined and studied as a class of emotions with positive valence (generally feeling good) such as joy, contentment, amusement, love and so on [9]. Admittedly, most research on emotion recognition in the field of affective computing mainly focused on affective dimensions such as valence and arousal of positive emotions rather than discrete emotion categories [10,11]. For example, the widely used emotion database such as DEAP was constructed based on valence and arousal dimensions [12]. One possible explanation is that different positive emotions correlate with each other highly on subjective feelings compared to negative emotions, which is difficult to distinguish from each other [13]. However, recent study found that self-reported emotional experience was better captured by emotion categories such as "amusement" which is more frequently used in daily life than by ratings of widely measured valence and arousal dimensions [14].

To sum up, the structure of extensive positive emotion categories on subjective feelings and standardized database specifically for positive emotions remain to be explored. The aim of this paper is to establish effective positive emotion-inducing material database and explore the structure of positive emotion categories, which could lay the foundation for further positive emotion recognition and analysis studies both from the perspective of theory and application.

\section{Literature REVIEW}

\subsection{Existing Positive Emotion Materials}

Various methods have been utilized to induce emotion through different types of materials such as words and texts [15], pictures(such as the International Affective Picture System, IAPS, [16]), sounds (such as the International Affective Digitized Sounds, IADS, [17]) and so on. 
Compared to other emotion-inducing materials, emotional video clips have been widely used in both psychological research $[18,19]$ and emotion recognition and analysis research [20,21]. One explanation is that film-induced emotional states overcome some of the fundamental reliability issues that arise from other emotional materials. Specifically, scenes from film clips are dynamic compared with static images or sounds, and using the film clips is a more realistic, easier to carry out task than the emotion imagination task [22].

The widely used databases for emotion recognition via physiological signals, such as MAHNOB-HCI [23], DREAMER [24], SEED [25] and AMIGOS [26] were constructed based on dimensional model of emotion, such as 2-D model (valence and arousal) or 3-D model (valence, arousal and dominance). And each positive emotion in these databases were all represented as a positive-valenced emotion rather than a certain positive emotion category. To the best of our knowledge, there's no existing standardized database especially for different categories of positive emotions. But some of the existing studies have explored this issue from different perspectives. First, according to the dimensional model of emotion, several emotion databases have been established such as DEAP [12], BAUM-2 [27] and so on. Additionally, researchers are beginning to use the film-induced EEG (electroencephalogram) signals to classify different positive emotions, most of them have focused on positive emotions with different arousal levels [28]. For example, Wang, Nie and Lu [29] have classified film-induced relax (low arousal) and joy (high arousal) states with an accuracy of $66.51 \%$ using the PSD (power spectral density) features extracted from EEG signals. A similar classification between relax and joy using EEG signals but different classifiers was also found in [30].

Notably, both the number of positive emotions and the selection of specific emotions in most of the above-mentioned studies were determined empirically. Therefore, it is questionable whether these studies could provide complete coverage of all possible positive emotions. What's more, it was shown that self-reported emotional experience was better captured by specific discrete emotions than by valence and arousal dimensions [14].

To address these issues, a recent EEG study [31] has adopted the ten positive emotion framework proposed by Fredrickson [7]. In their study, film clips were employed to elicit ten positive emotions which were further categorized into three clusters, based on the subjective ratings from the participants [31]. Further, the binary classification between higher and lower ratings on these positive emotions and the binary classification between three clusters achieved accuracies of approximately $80 \%$ using spectral power features extracted from EEG signals. Similar results were reported in their follow-up fNIRS (functional near-infrared spectroscope) study as well [32]. However, the emotion-inducing materials were not standardized and validated which were not enough to constitute a positive emotion database.

\subsection{Positive Emotion Categories}

Admittedly, as a class of emotion, positive emotions were difficult to distinguish from each other clearly from the perspective of category [9]. But some previous studies have found that different positive emotion categories such as awe, contentment, interest, love, and pride had respective cognitive, physiological, and behavioral characteristics which were closely related to particular functions distinctively $[33,34]$. Some researchers have made attempts to set up theoretical models of positive emotion categories. As proposed by Isgett and Fredrickson [5], ten representative positive emotions were frequently experienced in people's daily life, including joy, gratitude, serenity, interest, hope, pride, amusement, inspiration, awe and love. We introduced each positive category as follows.

People will experience joy when their current circumstances present unexpected good fortune [5]. However, the Chinese meaning of joy is not specific and is associated with several positive feelings simultaneously such as gratitude, pleasure and amusement to some extent [35], and therefore it was not considered in our proposed database. Gratitude is typically defined as an emotional state which emerges when someone has achieved unexpected good fortune and this positive outcome comes from another person [36]. Serenity is also called contentment, which is defined as the feeling of comfortable and satisfying towards people's current circumstances [4]. It is often achieved after satisfying one's own desires and different people have their own standards for their serenity. Because previous study reported that it was difficult to elicit general feeling of serenity using video clips [37], serenity was not considered in our proposed database as well. Interest is defined as a "knowledge-based" emotion which creates the urge to explore and learn novel information [38]. Hope, opposed to hopeless, arises in dire circumstances in which someone believes that something positive, which does not presently apply to one's life, could still materialize [39]. Pride is defined as a manifestation of a strong self that inspires a strong feeling similarity to others who are perceived strong, as well as a distinction from weak others [40]. Amusement as a typical positive emotion is frequently elicited by some nonserious social incongruity events that violate expectations [41]. Inspiration arises when people witness human excellence in some manner which motivate them to pursue personal growth [4]. Awe is a complex emotion which refers to an intense feeling of astonishment, wonder and connectedness that emerges when people encounter something (e.g., artistic works, scientific discovery, natural wonders) beautiful or powerful on a grand scale that transcends previous knowledge schemas [42, 43]. Love refers to the positive feeling that people experience in the context of a safe, interpersonal connection or relationship [4]. It is worthy of noting that love is a comprehensive emotion which can be divided into different types of love according to different types of attachments (e.g., maternal, romantic, and peer attachments in [44]). Therefore, these three types of love were considered simultaneously as a unified one in our study. Romance is experienced in a romantic relationship and motivates approach towards a romantic target [45]. Friendship refers to the friendly feeling and is frequently experienced and reported in daily life [46]. Tender- 
ness is characterized as a warm-and-fuzzy feeling corresponding to family love and attachment in present study.

In addition to these representative positive emotion categories, there are still other positive emotions worth being paid attention to. For example, it was recently found that video clips could reliably elicit 27 distinct varieties of reported emotional experience using self-report methods, among which some positive categories were not included in the representative categories mentioned above [14]. Aesthetic appreciation is defined as the emotion that emerges when someone perceives and evaluates a stimulus (such as art, literature, music, nature, or appealing sights and sounds) for its aesthetic appeal or virtues [47]. Sympathy is experienced when people witness other people or animal that is hurt or suffering, and is experiencing a current need [48], as a result of which it will lead to positive functions such as an approach orientation and facilitating pro-social behaviors [49]. Respect exhibits the endorsement that other person is an individual of honor and worth [50]. Adoration is a positive emotion towards excellence elicited by an outstanding person or object that cannot be fully attained by anyone else [51]. Craving is defined as the subjective sense of wanting and being motivated to approach a certain object (e.g., food, money), which is closely related to self-regulation [52]. Relief refers to the aversion of an undesired outcome [53].

Based on an extensive literature review, 16 categories of positive emotions were initially taken into consideration in our study: gratitude, interest, hope, pride, amusement, inspiration, awe, romance, friendship, tenderness, aesthetic appreciation, sympathy, respect, adoration, craving and relief. We aimed to establish effective eliciting materials of these 16 categories of positive emotions and explore the structure and framework of these categories.

\subsection{Autonomic Nervous System Features of Positive Emotion}

In addition to the most direct subjective feelings of different positive emotion categories, physiological characteristics also serve an important role in assessing and differentiating emotional states [20]. Emotion recognition using physiological features has raised great concern in the field of affective computing [54]. Specifically, emotion recognition using signals from the neural responses of human central nervous system (CNS) such as EEG has achieved great recognition accuracy [20]. However, most EEG signals could only be obtained in the laboratory due to low portability and inconvenience of data collection setting. With the development of wearable devices which can collect biosignals from autonomic nervous system (ANS, a part of peripheral nervous system), emotion recognition attempts to use signals from ANS which may further get higher applicability in real-world scenarios. Note that ANS were actually recorded in many of the EEG databases as well.

For example, a weightless and wearable electrocardiogram (ECG) device was created to collect ECG signals and two important physiological features extracted from ECG signals were reported to be most effective in differentiating sadness, anger, fear and happiness [55]. Furthermore, an overall accuracy for four-class recognition of amusement, anger, grief, and fear induced by film clips was achieved using fingertip blood oxygen saturation, galvanic skin response (GSR) and heart rate [56]. As for the valence and arousal dimensions, a framework to recognize valence and arousal in real time based on physiological signals collected from the pervasive wearable device was proposed and evaluated under a common environment. It showed accuracies of $75.57 \%$ for valence and $83.77 \%$ for arousal using random forest classification algorithm [57].

Although previous studies found that different positive emotions showed distinct physiological patterns [34], to the best of our knowledge, there is no study paid attention to recognize different positive emotion categories which are experienced more frequently and naturally in daily life using signals from ANS. If peripheral physiological features are able to differentiate different positive emotional states, wearable devices which can collect signals such as heart rate, heart rate variability, skin conductance, respiration rate, etc., should have a broader application prospect in the field of positive emotion analysis and interaction. Fortunately, some previous studies have demonstrated this hypothesis initially. Addressing the functional need for individuals under an evolutionary framework, a fivecategory positive emotion set was proposed (anticipatory enthusiasm, attachment love, nurturant love, amusement, and awe) and their possible neurophysiological differences were assessed [58]. Using static images to elicit these emotions, the multi-dimensional ANS signals indeed revealed significant distinct patterns.

Based on the findings of previous studies, one more purpose of our present study is to acquire peripheral physiological signals under positive emotional states through wearable smart devices. Our study focused on two primary signals, photoplethysmography (PPG) and GSR, which were reported to be sensitive to emotional changes $[59,60]$. Conveniently small, unobtrusive devices such as smartwatches are equipped with PPG sensor which can transmit specific light wavelengths into skin tissue, and measure the amount of light reflected back and thereby measure the changing volume of blood in the tissue over time following each pulse wave ejected from the heart [61]. On the other hand, GSR is the electrical conductance of the skin and depends on presecretory activity in the sweat glands [62].

\subsection{Contributions of Present Study}

Taken together, there are three major contributions of this paper. First, our constructed emotion database was based on a large sample (over 300 participants) compared to existing databases. Thus, individual differences of subjective feelings have been significantly reduced and the video clips were more representative. Second, more positive emotion categories were collected from a large online video clip pool in our constructed database than existing emotion databases. To construct such a database, the present study followed the approach outlined by Gross and Levenson [22] based on the discrete conceptualization of emotion with the aim of addressing as many discrete positive emotions as possible in the Chinese culture. What's more, the present study concentrated on the perceived 
emotion, which was previously confused with the conveyed emotion in most existing studies. Last but not the least, both the subjective feelings of different positive emotions and a physiological database corresponding to emotional video database were also established. The structure and framework of positive emotion categories according to subjective feelings were explored. Physiological signals from ANS when watching positive emotional video clips were also collected. Meanwhile, PPG and GSR feature system was established to recognize positive emotions. And the current CPED database will be publicly available for research on affective computing in the future.

The remainder of this paper is organized as follows. Section 3 presents the establishment of positive emotions database including the selection of video clips as emotioninducing materials and the acquisition of PPG and GSR signals when individuals were watching these emotional materials. Sections 4 and 5 present behavioral results and physiological features, respectively. Section 6 presents the discussion and Section 7 offers the concluding remark.

\section{Construction of a Standardized Chinese Positive EMotion DATABase}

\subsection{Primary Selection}

As summarized in Section 2.2., 16 categories of positive emotions were initially taken into consideration in our study: gratitude, interest, hope, pride, amusement, inspiration, awe, romance, friendship, tenderness, aesthetic appreciation, sympathy, respect, adoration, craving and relief. Ten research assistants were invited to select film clips that were representative of these 16 emotion categories from online film websites (e.g., douban, Mtime). They were first trained to be familiar with the definition, the inducing situation and the subjective feelings of different positive emotion categories based on the theoretical psychological knowledge.

According to [9], different core relational themes (CRTs) which represent the prototypical "script" associated with an emotion experience of positive emotions were captured. These CRTs were adopted in our study to guide the initial selection of emotion-inducing situation that was displayed in the video clips. For example, "aware of incongruity" and "playful with others" were reported to be two core relational theme items of amusement, and then a clip which describes a group of male friends dressed up as female nurses and sneaked into the hospital to cooperate in solving a case would be suitable for eliciting individual's amusement emotion. Similarly, "situation challenged worldview" and "felt small relative to other" were characterized as two core relational theme items of amusement, and then a clip depicts the comparison of the size between galaxies and the earth in the universe was selected as candidate video clip to induce awe emotion initially. Meanwhile, "attention absorbed by novelty" as well as "felt they could explore" were shown to be two core relational theme items of interest, and thus, a video clip introduces seven of the most exotic places in the world would provide novelty for the viewers and stimulate their desire to explore. Moreover, two core relational theme items of gratitude were "felt benefited by another's action" and "wanting to give back", and then a public service announcement which tells several stories about those ordinary little warm segments in life and good will from strangers would meet the requirements. As for the emotion categories which were not included in [9], video clip selection criteria were based on the definition of the corresponding emotion.

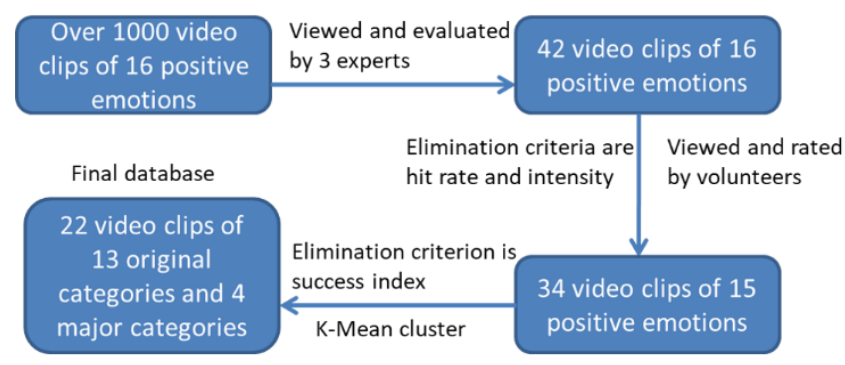

Fig. 1. The screening process of current positive emotional video clip database.

Ten well-trained research assistants were instructed to select and extract the video clips that were easy to understand and were effective for eliciting at least one of these positive emotions. All the selected film clips lasted for 1 to 3 minutes with relatively consistent and integrated content from film or online video websites. Over 1000 video clips were initially selected. And these ten assistants were asked to give an intensity score (from 1 to 9) on target emotion of each video clip. The Kendall's W of these ten assistants' rating was 0.75 , which showed relatively high consisitency among ten raters. Afterwards, three experts with previous experiences in emotion assessment were invited to screen all these film clips for their intelligibility (viewers can easily understand the thematic contents without additional explanation) and discreteness (they were likely to elicit a specific reported emotional state) [37]. Finally, 42 video clips were selected and each of them was selected with the consent of at least two of the three experts. All these 42 film clips were named as emotional categories and numbered such as "awe-01", "interest-02" and so on. The screening process of video clips was presented in Fig. 1.

\subsection{Experiment for Further Evaluation}

\subsubsection{Participants}

A total of 319 undergraduate students and graduate students participated in the current experiment, seven of them were excluded due to their casual response and incomprehension of contents of video clips according to subjective ratings and detection questions. Finally, a sample of 312 people was obtained (156 males and 156 females), whose average age was 21.6 years (range $=18-30, \mathrm{SD}=2.2$ ). All the participants were healthy without reported emotional problems recently such as depression and anxiety which might influence experimental results. The Ethical approval was supported by the Institutional Review Board (IRB) of the Institute of Psychology, Chinese Academy of Sciences. All the participants agreed to take part in the experiment willingly. They were told the entire procedure of the ex- 
periment and had the right to withdraw from the experiment at any time.

\subsubsection{Experimental Procedure and Behavioral Measures}

The experiment was carried out by several people in subgroups (1 to 7 participants) in a quiet room. Each participant was equipped with a keyboard, mouse and soundproof headset to watch the video clips separately and make a response after watching each video clip. The film clips were displayed on a 15-inch color liquid crystal display screen which was placed 70 centimeters above the ground. The participants were separated by a clapboard and they were asked to watch the video clips carefully without talking or influencing others.

Upon arrival, participants were asked to have a short break to calm their minds and wait till everyone in the subgroup arrived. The experimenter explained the whole procedure of the experiment and what the participants should do during the whole experiment to make sure that all the participants understand experimental intentions as well as some key options and considerations. After that, participants signed informed consents and fulfilled a short basic information questionnaire (such as age and gender) online. Then, each participant wore the smart wristwatch with the help of experimenters. The participants were asked to keep watch-wearing hands free from strenuous exercise during watching video clips.

A practice trial was arranged before formal watching and evaluations in order to make participants familiar with the experimental procedure. Then the video clips were presented sequentially to participants. The 42 film excerpts were pseudo-randomly assigned to four subgroups (each subgroup contains 11 or 12 video clips). Within these subgroups, video clips belonging to the same category were not presented continuously. Each participant was randomly assigned to one of the groups. The number of participants in the four subgroups was balanced in total. The gender was balanced for each film clip as well. Two participants with adjacent seats were arranged to watch video clips of different subgroups.

Before watching each video clip, participants were asked to complete an operation of adding and subtracting twelve numbers as a distraction task to eliminate the effect of the previous film clip on the current one. They were asked to enter the answer of the operation in the answer box on the screen (each person was assigned a pen and paper for calculation). Then they were instructed to rest for half a minute quietly in order to record baseline data and make preparations for watching next video clip. A beep sound indicated the end of the rest. If participants were ready, they could press the "next" button on the screen to watch the video clip.

After each video clip, participants were asked to rate the intensity of each target emotion (the emotion category that the corresponding film clip aims to elicit) using Emotion Evaluation Scale (EES) based on previous studies [22, 63].
A total of 16 items corresponding to 16 target positive emotion categories (gratitude, interest, hope, pride, amusement, inspiration, awe, romance, friendship, tenderness, aesthetic appreciation, sympathy, respect, adoration, craving and relief) constitute the scale. The participants were asked to evaluate the intensity of their subjective emotional feelings on all items after viewing each film clip on a 9point scale $(1=$ "not at all", $9=$ "extremely"). The instruction of this EES were "Please choose the intensity of each of the following emotions based on your current feelings" and "Please make sure that the emotion intensity is based on your own subjective feelings other than the mood of the protagonist in the video", and "No matter you know what emotion this video is trying to convey or not, you only evaluate whether you actually experience each of the following emotions".

After scaling the emotion evaluation, a detection question was presented to participants in order to check whether they have understood the plot of the video clip or watched the clip carefully. This question required the participants to indicate what content of video clips made them feel the emotion of the moment.

Then, the next trail would repeat the above process until the experiment was over. The whole experiment would last for approximate two hours. Laboratory settings and the experimental procedure for watching video clips see Fig. 2.

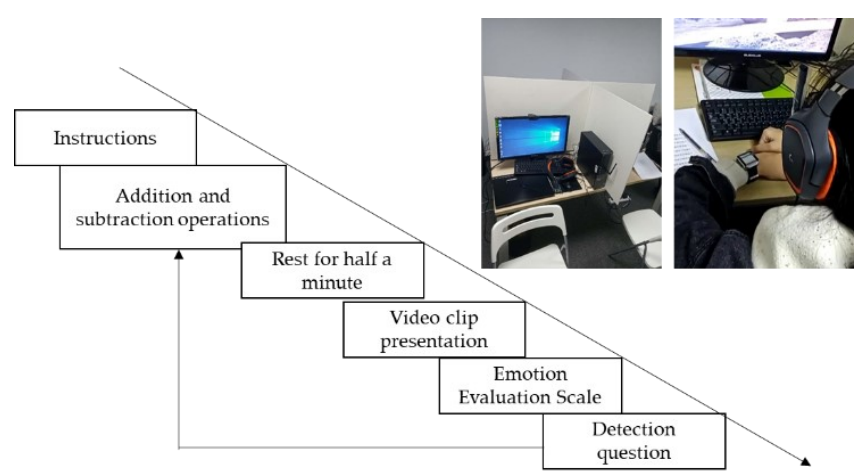

Fig. 2. Laboratory settings in our study and the timing diagram of the experiment.

\subsubsection{Physiological Signals Acquisition and Feature Extraction}

To obtain GSR and PPG signals, the participants wore customized designed wristbands (Psychorus, China) on their subdominant hands when they watched and rated video clips. GSR signals were acquired by surface electrodes with conductive gels at a sampling rate of $40 \mathrm{~Hz}$ with a resolution of $0.01 \mu \mathrm{S}$. The precision HRs were collected using the photoplethysmography (PPG) method. Green light of 532 $\mathrm{nm}$ wavelengh was used and the reflected light was measured at a sampling rate of $20 \mathrm{~Hz}$. Three-axis accelerations were recorded at $20 \mathrm{~Hz}$ as well with a precision of $1 / 2048 \mathrm{~g}$ [64], but not used in the present study.

GSR signals in the acquisition process can easily be contaminated with noise signals. We preprocessed the data by the removal of noises in the process of GSR recording to 
retain the effective data segments. Continuous GSR data are filtered with an empirical iterative algorithm (EIA) filter to minimize the introduction of noises [65]. Then, GSR signals were segmented into a number of 5-s time windows with an $80 \%$ overlap between two successive time windows. In each time-window, 90 GSR features were extracted (see Table 1), for example, the mean of the GSR data measured in units of micro-Siemens (i.e., average GSR) and the mean of the first-order difference of signals (i.e., average 1st-order derivative of GSR). Finally, to reduce the differences in signal characteristics caused by individual variability, we subtracted the baseline mean from each segment of the GSR signal and then mapped into the range [0, $1]$.

For PPG signals, we chose a 3rd-order bandpass Butterworth filter with a lower cutoff frequency of $0.5 \mathrm{~Hz}$ and a higher cutoff frequency of $8 \mathrm{~Hz}$ to maximize the retention of the original signals [66]. Similarly, a 5-s sliding window with an $80 \%$ overlap between two consecutive windows was used to segment the filtered and noise-free PPG data. 23 features were extracted from PPG signals in each window (see Table 1), for example, the mean of PPG data measured in units of micro-Volts (i.e., average PPG) and the mean of all instantaneous heart rates measured in units of beat per minute (i.e., average heart rate) which is based on the duration of PPG waveform produced by two heartbeats as a unit time).

Table 1 Features extracted from PPG and GSR signals

\begin{tabular}{|c|c|}
\hline Signal & Extracted features \\
\hline $\begin{array}{c}\text { GSR } \\
(\mathrm{n}=90)\end{array}$ & $\begin{array}{l}\text { mean, median, standard deviation, minimum } \\
\text { and maximum of the first difference, second } \\
\text { difference and GSR signal, mean and standard } \\
\text { deviation of the first order derivative and sec- } \\
\text { ond order derivative, proportion of negative } \\
\text { derivation in the first order derivative and sec- } \\
\text { ond order derivative, number of local minima, } \\
\text { number of local maxima, skewness, kurtosis, } \\
\text { root mean square, mean resolute, mean abso- } \\
\text { lute deviation, the first degree difference di- } \\
\text { vided standard deviation and second degree } \\
\text { difference divided standard deviation of GSR } \\
\text { signal, spectral power in the [0-2.4] Hz band, } \\
\text { spectrogram, power spectral density }\end{array}$ \\
\hline $\begin{array}{c}\text { PPG } \\
(\mathrm{n}=23)\end{array}$ & $\begin{array}{l}\text { heart rate, rising and falling time of the PPG } \\
\text { signal, rising and falling height of the PPG sig- } \\
\text { nal, cardiac period, average and standard de- } \\
\text { viation of PPG, energy of PPG, time duration } \\
\text { in time domain, bandwidth in frequency do- } \\
\text { main, time-bandwidth product, dimensional- } \\
\text { ity of the PPG signal, average and standard de- } \\
\text { viation of heart rate variability in the fre- } \\
\text { quency bands ([0.003- } 0.04] \mathrm{Hz},[0.04-0.15] \mathrm{Hz} \text {, } \\
[0.15-0.4] \mathrm{Hz}) \text {, energy in the frequency bands } \\
([0.003-0.04] \mathrm{Hz},[0.04-0.15] \mathrm{Hz},[0.15-0.4] \mathrm{Hz}) \text {, } \\
\text { signal ratio between the frequency bands }[0.04- \\
0.15] \mathrm{Hz} \text { and }[0.15-0.4] \mathrm{Hz}\end{array}$ \\
\hline
\end{tabular}

\subsubsection{Data Analysis Method}

For the behavioral results, all the data processing was done through $\mathrm{R}$ language. First, hit rate and intensity for each video clip were computed as previous study $[22,37]$ which were firstly examined to exclude video clips with poor inducing effect. Hit rate is defined as the proportion of reviewers who rate the target emotion score at least one point higher than the non-target emotion. Intensity is the mean level at which the target emotion was rated. Then, Pearson correlations were calculated among initially selected emotion categories and the $p$-values were corrected according to the false discovery rate (FDR) using Benjamini and Hochberg's (BH) method [67].

After that, the k-means clustering algorithm was adopted in the current study to perform systematic cluster analysis on all video clips with subjective ratings of each emotion category from participants as input. The step of kmeans clustering is to randomly select $\mathrm{k}$ objects as initial clustering centers, then calculate the distance between each object and each seed clustering center, and assign each object to the nearest clustering center. Cluster centers and objects assigned to them will be represented as a cluster. Then the clustering center of the cluster is recalculated according to the existing objects in the cluster. This process will be repeated until a termination condition is met (for example, the within cluster sum of squares is the smallest) [68].

A challenge in k-means clustering is how to select the optimal number (k) of clusters. In this study, we applied the NbClust package in R [69], which can provide 30 indexes for determining the optimal number of clusters in a data set and propose the best clustering scheme from the different results obtained by varying all combinations of number of clusters, distance measures (such as "euclidean", "maximum", "manhattan", "canberra", "binary", "minkowski"), and clustering methods (such as "ward.D", "ward.D2", "single", "complete", "average", "mcquitty", "median", "centroid", "kmeans"). Here we chose "kmeans" as clustering method and "euclidean" as distance. The details and computing methods of all the 30 indexes (containing " $\mathrm{CH}$ index"," DB index","Duda index"," Rubin index","Gamma index","Hubert index"and so on) provided by NbClust can be seen from [69], which would be cumbersome to present them concretely in the current section. For example, The Davies and Bouldin index (DB index) is calculated as the ratio of the sum of within-cluster scatter to between-cluster separation and the Rubin index is a function of the ratio of the determinant of the total sum of squares and cross products matrix to the determinant of the pooled within cluster matrix. And origin emotion subcategories can be clustered into several reasonable major categories according to the result of NbClust. And each origin emotion category will belong to a major emotion category after clustering.

Then, success index was computed by following previous research to select the best video clips into the final database after clustering analysis [37]. Success index was defined as the sum of two z-scores derived from normalizing the hit rate and the intensity. For the finally selected video clips, a series of one-way ANOVAs were conducted to examine the differences in the ratings between target emotion of major category and non-target major category to 
conduct final verification. And demographic differences were also tested on the behavioral data of the final selected video clips.

As for PPG and GSR features, all the features were entered into classifier to test the possibility of recognizing positive emotions using PPG and GSR signals. But not all of these features are requisite to be used for classification. To select useful features, Linear Discriminant Analysis (LDA) was applied [68], which projected the original samples to a lower-dimensional sample space for simultaneously select best features and achieve the best classification performance.

After selecting useful features by LDM, these features are input into a widely used classifier called support vector machine (SVM) to classify four major positive emotion categories; that is, fun, empathy, creativity and esteem. The original SVM is a typical two-class classifier, that is, it only answers samples that belong to a positive or negative class. To achieve a multiclass classification, we built a classifier between every two classes, a total of six SVMs. In our implementation using Python, we applied scikit-learn for LDA and SVM with a RBF kernel function. We optimized the cost parameter $c$ and the gamma using grid search. And the 10-fold subject-independent cross validation was utilized to evaluate the performance of recognition.

\section{BehaVioral Results}

\subsection{Hit Rate and Intensity}

First, according to the hit rate, four video clips were removed from the database due to low hit rate including gratitude-02 (1.30\%), relief-01 (1.28\%), relief-02 $(2.53 \%)$ and relief-03 (4.00\%).

Second, according to the result of intensity, several video clips were also removed from the database because of low intensity or a lower rating for target emotion than non-target emotion. Specifically, amusement-04 (5.45) and hope-02 (3.33) were removed due to low intensity scores. Sympathy-01 was removed because the rating for sympathy (5.50) was lower than tenderness (6.13). Similarly, interest- 03 was also removed from further analysis because the rating for interest (4.82) was lower than aesthetic appreciation (5.96). Finally, 34 video clips that met the initial standards of our database were selected.

\subsection{Correlations among Positive Emotion Categories}

Although a total of 15 discrete positive emotion categories (note that all the video clips of relief were removed) were considered, the subjective feelings of different positive emotions were highly correlated [13], as a result of which it will lead to low hit rate and similar intensity. For example, when people respect a national hero, they may be proud that their country has such a hero. But respect and pride are two discrete positive emotions. Thus, Pearson correlations were calculated among 15 positive emotion categories.

There were significant correlations between the majorities of positive emotion categories (see see Table 2). For example, respect, craving, adoration, amusement, hope and gratitude were significantly correlated with each other ( $p s$ $<.01)$. In addition, it was found that awe was significantly correlated with other positive categories ( $p s<.01)$ except romance and friendship. Pride was significantly correlated with other positive categories ( $p s<.01$ ) except romance. Apart from that, romance was significantly correlated with other positive categories ( $p s<.01$ ) except sympathy, respect and adoration. Except for this, inspiration was significantly correlated with other positive categories $(p s<.01)$ except amusement. Interest was significantly correlated with other positive categories ( $p s<.01$ ) except sympathy. Sympathy was significantly correlated with other positive categories $(p s<.01)$ except sympathy.

As for the coefficient size and direction of correlation, visualization was performed with an $\mathrm{R}$ package called corrplot which could provide a method of reordering correlation matrices and displaying saliency levels with color in correlation graphs [70]. It can be seen from Fig. 3 that some structural relationship may exist within the correlations among these positive emotion categories, which was reflected in the fact that similar colors could form larger color blocks. Thus, a cluster analysis was performed to explore the structure of positive categories based on subjective feelings reported by participants.

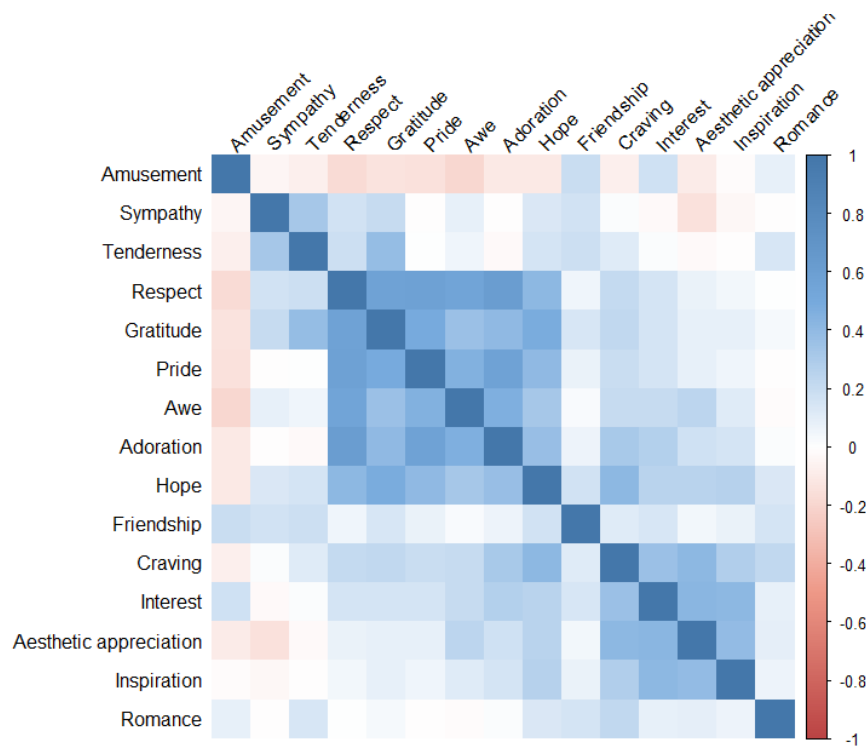

Fig. 3. The size and direction of the correlation between positive categories were represented by color. Blue represents positive correlation, red represents negative correlation, and the deeper the color, the higher the degree of correlation. 
Table 2 Pearson correlation coefficient and significance $\left({ }^{* *} \mathrm{p}<.01,{ }^{*} \mathrm{p}<.05\right)$ among positive emotion categories

\begin{tabular}{|c|c|c|c|c|c|c|c|c|c|c|c|c|c|c|c|}
\hline & 1 & 2 & 3 & 4 & 5 & 6 & 7 & 8 & 9 & 10 & 11 & 12 & 13 & 14 & 15 \\
\hline 1 Tenderness & 1 & & & & & & & & & & & & & & \\
\hline 2 Awe & $.053^{* *}$ & 1 & & & & & & & & & & & & & \\
\hline 3 Pride & .001 & $.456^{* *}$ & 1 & & & & & & & & & & & & \\
\hline 4 Romance & $.141^{* *}$ & -.020 & -.001 & 1 & & & & & & & & & & & \\
\hline 5 Inspiration & -.008 & $.115^{* *}$ & $.058^{* *}$ & $.063^{* *}$ & 1 & & & & & & & & & & \\
\hline 6 Friendship & $.180^{* *}$ & .029 & $.077^{* *}$ & $.157^{* *}$ & $.074^{* *}$ & 1 & & & & & & & & & \\
\hline 7 Interest & .019 & $.207^{* *}$ & $.152^{* *}$ & $.081^{* *}$ & $.413^{* *}$ & $.145^{* *}$ & 1 & & & & & & & & \\
\hline 8 Sympathy & $.321^{* *}$ & $.089^{* *}$ & -.001 & -.004 & $-.038^{*}$ & $.167^{* *}$ & -.026 & 1 & & & & & & & \\
\hline 9 Aesthetic & -.027 & $.240^{* *}$ & $.089^{* *}$ & $.092^{* *}$ & $.391^{* *}$ & $.043^{*}$ & $.425^{* *}$ & $-.143^{* *}$ & 1 & & & & & & \\
\hline 10 Respect & $.188^{* *}$ & $.554^{* *}$ & $.587^{* *}$ & .007 & $.043^{*}$ & $.058^{* *}$ & $.156^{* *}$ & $.168^{* *}$ & $.072^{* *}$ & 1 & & & & & \\
\hline 11 Craving & $.112^{* *}$ & $.209^{* *}$ & $.195^{* *}$ & $.226^{* *}$ & $.289^{* *}$ & $.110^{* *}$ & $.360^{* *}$ & .013 & $.419^{* *}$ & $.210^{* *}$ & 1 & & & & \\
\hline 12 Adoration & -.030 & $.465^{* *}$ & $.577^{* *}$ & .019 & $.160^{* *}$ & $.064^{* *}$ & $.273^{* *}$ & -.004 & $.170^{* *}$ & $.616^{* *}$ & $.311^{* *}$ & 1 & & & \\
\hline 13 Amusement & $-.078^{* *}$ & $-.192^{* *}$ & $-.141^{* *}$ & $.081^{* *}$ & -.011 & $.198^{* *}$ & $.174^{* *}$ & $-.046^{* *}$ & $-.100^{* *}$ & $-.174^{* *}$ & $-.078^{* *}$ & $-.108^{* *}$ & 1 & & \\
\hline 14 Hope & $.153^{* *}$ & $.322^{* *}$ & $.409^{* *}$ & $.132^{* *}$ & $.264^{* *}$ & $.165^{* *}$ & $.258^{* *}$ & $.131^{* *}$ & $.250^{* *}$ & $.414^{* *}$ & $.415^{* *}$ & $.372^{* *}$ & $-.101^{* *}$ & 1 & \\
\hline 15 Gratitude & $.386^{* *}$ & $.369^{* *}$ & $.493^{* *}$ & $.039^{*}$ & $.086^{* *}$ & $.145^{* *}$ & $.158^{* *}$ & $.203^{* *}$ & $.086^{* *}$ & $.579^{* *}$ & $.220^{* *}$ & $.401^{* *}$ & $-.131^{* *}$ & $.484^{* *}$ & 1 \\
\hline
\end{tabular}

\subsection{Systematic cluster analysis}

NbClust package was utilized to determine the best number of clusters of these 34 video clips in 15 positive emotion categories [69]. The results were showed in Fig. 4, showing that clustering into four categories was the most appropriate, because there were up to six indexes supporting clustering into four categories, which was the largest number of indexes.

Then the k-means clustering with the number of clusters set to 4 was performed, leading to four major categories created. The results revealed that amusement-01, amusement-02, amusement-03, friendship-01 and friendship-02 were grouped into cluster- 1 . Tenderness-01, tenderness-02, gratitude-01, romance-01, hope-01, hope-02, sympathy-02 and sympathy-03 were grouped into cluster2. Awe-01, awe-02, awe-03, inspiration-01, inspiration-02, inspiration-03, aesthetic appreciation-01, interest-01, interest-02, interest-04, craving-01, craving- 02 , craving-03 and adoration-01 were grouped into cluster-3. And respect-01, respect- 02 , pride- 01 , pride- 02 , pride- 03 as well as pride- 04 were grouped into cluster- 4 .

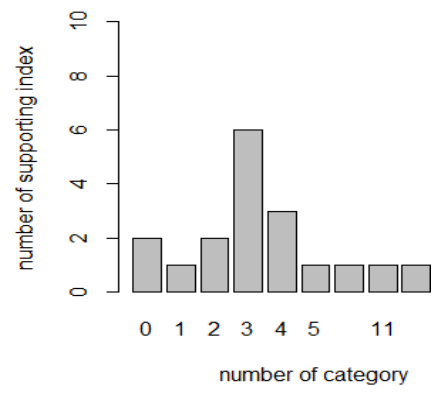

Fig. 4. The result of Nbclust showed that clustering into four categories had most supporting indexes.
As shown in Fig. 5, four centroids were dispersed. Moreover, video clips belonging to the same emotion category were clustered into the same bigger category at the same time. Therefore, the current results of clustering were reasonable. In terms of theoretical expectations, positive emotion categories in the cluster-1 can be defined as "fun", which is more related to funny things happening to people. Positive emotion categories in the cluster- 2 can be named as "empathy", which represents a large group of emotions to experience the feelings of others. The positive emotion categories in the cluster- 3 are related to inspiration and creation, in which people can see a broader world. The cluster-3 can be named as "creativity". As for positive emotion categories in the cluster-4, they represent the value evaluation and affective preference for both other people and self, which can be named as "esteem". From this perspective, the clustering results also accorded with the theoretical expectations.

\subsection{Success Index and final database}

Since each video clip was clustered into a new major category, both the hit rate and the intensity were recalculated. Then the new target emotion was reset to the mean of each original subcategory contained under the major category. For example, the target emotion of fun was computed as the average ratings of amusement and friendship, and the target emotion of esteem was computed as the average ratings of respect and pride. Then the success index was computed within each major category to select best video clips under each major category. Finally, video clips with success scores greater than zero could enter the database. And a total of 22 video clips from four major positive emotion categories formed final database (see Appendix). 


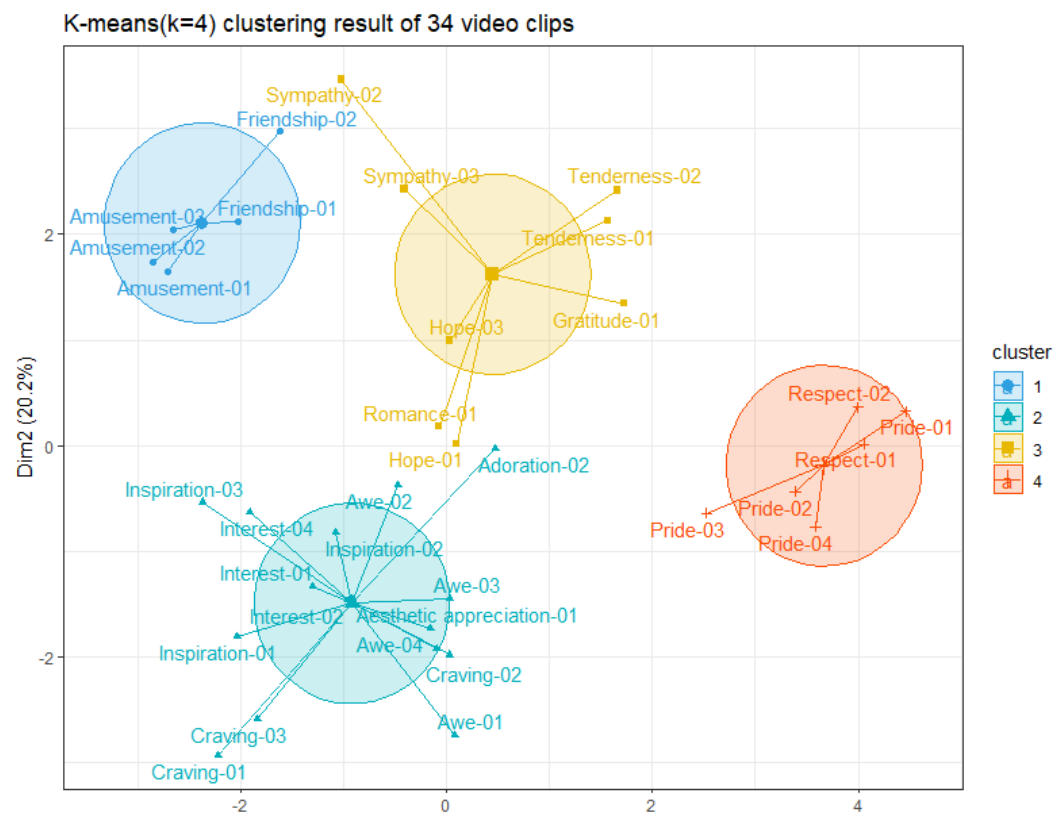

Fig. 5. The result of systematic cluster analysis (K-mean) according to subjective ratings on positive emotion categories. 34 video clips were clustered into four clusters which had the most supporting index.

For video clips in the final database, all video clips were rated significantly higher on target emotion than non-target emotion $(p s<.001)$ for the final database, which could indirectly reflect the validity of clustering and the independence of four major categories.

And for the gender difference, results did not reveal significant differences between men and women in their mean ratings of both original and major category target emotions for most video clips. Only two video clips showed significant gender differences. Men reported more amusement than women in response to amusement- 01 ( $p$ $=.029)$, whereas women reported more amusement than men in response to amusement-03 $(p=.035)$. Men also reported more fun than women in response to amusement$01(p=.016)$. As for the difference between participants majoring in arts or science, no significant differences were found for most video clips as well. Resulted only indicated that participants who majored in science reported more awe than those who majored in arts $(p=.026)$.

\section{Physiological features of final database}

A total of 113 features were extracted from PPG and GSR signals by following previous studies [56, 71]. For some basic features such as average heart rate, average PPG, average GSR, and average 1st-order derivative of GSR (see Appendix), demographic differences were firstly tested on these physiological features of each video clip in the final database. For the gender difference, the average heart rate of men was significantly lower than women $(p=.017)$ during their watching of amusement-01. The average heart rate of men was significantly lower than women $(p=.038)$ during their watching of craving-03. Apart from that, no significant differences were found between men and women on physiological features. As for the difference be- tween participants majoring in arts or science, no significant differences were found for all the video clips in the final database.

Second, the time series of four major emotion categories were also presented in Fig. 5. Since the plot of the movie is progressing with time, the inducing effect of emotion is also accumulated with time. Usually the intensity of the viewer's emotional feelings reaches a climax at the end of the emotional video. Thus, within each major category, the physiological data of all videos in the last 50 seconds was averaged to explore the difference between four categories. For the average heart rate, it can be seen from Fig. 6 that the creativity and esteem category were higher than fun and the creativity category showed greater variability than other emotion categories during last 50 second of video clips.

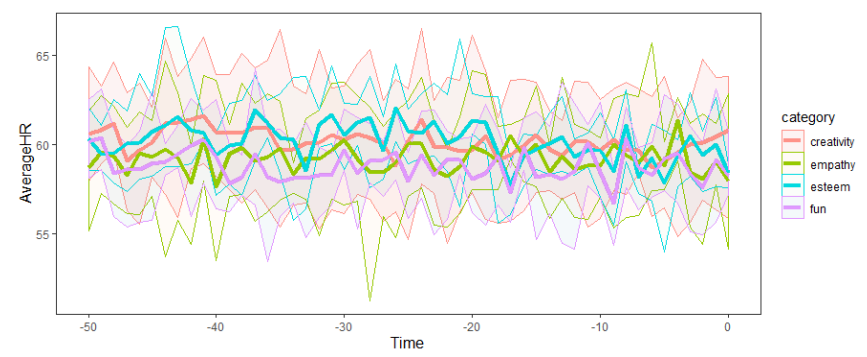

Fig. 6. The time series of average heart rate of four positive emotion categories (the shadow areas represent the range between maximum and minimum values).

The data set of 309 (10 out of 319 physiological sample were excluded due to recording error) participants were randomly partitioned into 10 subsets, one for 30 participants. Of the 10 subsets, a single subset was retained as the validation data for model testing, and the remaining 9 sub- 
sets were used as training data. The procedure was repeated 10 times, with each of the 10 subsets used exactly once as the validation data. Then the average classification accuracy $(45.84 \%)$ was computed as a baseline.

\section{Discussion}

The presented study established a video clip-based database for positive emotion analysis through an emotional video clip evaluation experiment. Both subjective rating scores of target emotion and objective peripheral physiological data (PPG and GSR) were recorded. There are two major contributions of the presented study. First, effective eliciting video clips specifically for positive emotions were established based on a large sample size. Subjective rating data collected from over 300 participants showed that highly correlated positive emotion categories could be clustered into four major positive categories (fun, empathy, esteem and creativity), which classified the structure of positive emotions systematically. Second, a peripheral physiological database corresponding to video clip database was also created for further emotional analysis.

For the original positive emotion categories, our database is better than existing databases from two perspectives. On one hand, a total of 15 original categories were eventually selected in the presented database (awe, gratitude, tenderness, friendship, romance, sympathy, pride, inspiration, interest, aesthetic appreciation, respect, adoration, craving, hope, and amusement), which was larger than positive emotion categories in previous databases [31, $35,37]$. This larger number indicated the possibility of inducing more discrete positive emotional categories using video clips. On the other hand, our criteria for screening video materials are more stringent than positive emotioninducing materials that used in previous study [31] based on hit rate, intensity and success index. What's more, only two amusing clips in the presented database showed demographic differences in subjective ratings, which reflected the universality of our materials. This difference might be due to the fact that female audiences prefer male protagonists while male audiences prefer female protagonists. As for awe, many magnificent landscapes appear in the video clips, and arts students have lower intensity of feelings for this kind of scene compared with science students. It might be due to the fact that arts students have a deeper understanding of beauty than science students.

However, it has to be pointed out that certain positive emotional state is still not suitable to be induced by video materials, such as relief. The possible explanation is that relief often occurs after a painful experience, so most of the time the video clips viewed by participants were to induce negative emotional experience. Meanwhile the final episode that intended to induce relief was very short.

Since most of the existing positive emotional materials were designed for Western, especially for English-speaking participants, the common emotional feelings among different cultures were considered in the presented database. Most of the films selected in current database are in Chinese. Such a comprehensive standardized database of positive emotional film clips could reflect East Asian cultures to some extent.

For the structure of positive emotion categories, our result found that four categories were the best structure according to the subjective feelings. Although it's a datadriven process, the result of clustering was in line with the theoretical expectations to some extent. However, this four-category positive emotion system was different from the three-category result reported by previous study [31]. In their study, according to the EEG response, different positive emotions were clustered into three major categories, as 'encouragement' for awe, gratitude, hope, inspiration, pride, 'playfulness' for amusement, joy, interest, and 'harmony' for love, serenity [31]. Only 'playfulness' category was similar to the 'fun' category in current database. Since both subjective feeling and physiological arousal are important components of emotional experience. This result might indicate that the subjective experience and the central nervous system response of intensive positive emotions are separated. To be specific, those positive emotions that share similar subjective feeling may have different neurological response. We will explore this direction in future studies. Nevertheless, the presented study still provides implications for labeling positive emotions more accurately using both original categories and higher-level major category, since subjective feelings are the most direct for emotional experiencers.

Last but not least, previous study [72] has found that different positive emotions can be represented by facial expressions and it showed that smiles and laughs occurred in all 16 positive emotions proposed by Ekman [73] which was different from the presented study. The presented study found that positive emotions could also be represented by peripheral physiological signals, which provided the possibility of positive emotion recognition using multi-modal signals. Accordingly more features can be extracted based on the presented database and input into more appropriate classifiers to establish a real-time positive emotion recognition system in future studies. It should be noted that positive emotions are correlated with each other in terms of subjective ratings and physiological response according to the current results, which brings forward higher requirements for the recognition of positive emotions.

\section{CONCLUSION}

In this paper we present a comprehensive study to establish a Chinese positive emotion database (CPED). Our study showed that positive emotions were highly correlated and could be clustered into four major categories (empathy, creativity, fun and esteem) based on the participants' subjective feelings. A total of 22 video clips that can successfully elicit these four major positive emotion categories were selected to form CPED database. PPG and GSR signals were collected when participants were watching video clips. As a baseline evaluation, a SVM classifier achieved a $45.84 \%$ accuracy for four-category recognition using PPG and GSR features. The advantage of CPED is able to elicit more positive emotions using representative 
video clips with smaller individual differences of subjective feelings than the existing databases. With these standardized positive emotion-inducing materials, physiological signals (e.g., EEG) and behavioral data (e.g., facial expression) could be collected to investigate the underlying neural and behavioral mechanisms of positive emotion categories and to develop a positive emotion recognition system for further emotion analysis and interaction.

\section{ACKNOWLEDGMENT}

This work was supported in part by the National Key Research and Development Plan (2016YFB1001200) and the National Natural Science Foundation of China (Grant No. 31771226, U1736220).

\section{REFERENCES}

[1] D. Keltner, and P. Ekman, "Facial Expression of Emotion," Handbook of emotions (second edition), M. Lewis and J. M. Haviland-Jones, eds., pp. 236-249: Guilford Publications, 2000.

[2] E. Diener, "Subjective well-being. The science of happiness and a proposal for a national index," American Psychologist, vol. 55, no. 1, pp. 34, 2000.

[3] M. E. P. Seligman, and M. Csikszentmihalyi, "Positive Psychology," American Psychologist, vol. 55, no. 2, pp. 514, 2000.

[4] B. L. Fredrickson, "Positive Emotions Broaden and Build," Advances in Experimental Social Psychology, E. Plant and P. Devine, eds., pp. 1-53, Amsterdam: Elsevier, 2013.

[5] S. F. Isgett, and B. L. Fredrickson, "Broaden-and-Build Theory of Positive Emotions," American Psychologist, vol. 359, no. 1449, pp. 1367-1377, 2004.

[6] N. S. Yegiyan, and A. P. Yonelinas, "Encoding details: positive emotion leads to memory broadening," Cognition \& Emotion, vol. 25, no. 7, pp. 1255-1262, 2011.

[7] B. E. Kok, K. A. Coffey, M. A. Cohn, L. I. Catalino, T. Vacharkulksemsuk, S. B. Algoe, M. Brantley, and B. L. Fredrickson, "How positive emotions build physical health: perceived positive social connections account for the upward spiral between positive emotions and vagal tone," Psychological Science, vol. 24, no. 7, pp. 1123-32, Jul 1, 2013.

[8] G. Veronese, M. Natour, and M. Said, "Positive emotions and life satisfaction in Palestinian children growing up amid political and military violence: a pilot study," Lancet, vol. 380, no. Suppl 1, pp. S16-S16, 2012.

[9] B. Campos, M. N. Shiota, D. Keltner, G. C. Gonzaga, and J. L. Goetz, "What is shared, what is different? Core relational themes and expressive displays of eight positive emotions," Cognition \& Emotion, vol. 27, no. 1, pp. 37-52, 2013.

[10] G. Valenza, A. Lanata, and E. Scilingo, "The Role of Nonlinear Dynamics in Affective Valence and Arousal Recognition," IEEE Transactions on Affective Computing, vol. 3, no. 2, pp. 237-249, 2012.

[11] X. Hu, J. Chen, F. Wang, and D. Zhang, "Ten challenges for EEG-based affective computing," Brain Science Advances, 2019.
[12] S. Koelstra, C. Muhl, M. Soleymani, J. S. Lee, and I. Patras, "DEAP: A Database for Emotion Analysis Using Physiological Signals," IEEE Transactions on Affective Computing, vol. 3, no. 1, pp. 18-31, 2012.

[13] L. F. Barrett, J. Gross, T. C. Christensen, and M. Benvenuto, "Knowing what you're feeling and knowing what to do about it: Mapping the relation between emotion differentiation and emotion regulation," Cognition \& Emotion, vol. 15, no. 6, pp. 713-724, 2001

[14] A. S. Cowen, and D. Keltner, "Self-report captures 27 distinct categories of emotion bridged by continuous gradients," Proceedings of the National Academy of Sciences of the United States of America, vol. 114, no. 38, pp. E7900, 2017.

[15] M. M. Bradley, and P. J. Lang, "Affective Norms for English Text (ANET): Affective ratings of text and instruction manual," Techical Report. D-1, University of Florida, Gainesville, FL, 2007.

[16] P. Lang, M. Bradley, and B. Cuthbert, International affective picture system (IAPS): affective ratings of pictures and instruction manual. University of Florida, Gainesville, Tech Rep A-8, 2008.

[17] M. Bradley, and P. J. Lang, The International affective digitized sounds (IADS): stimuli, instruction manual and affective ratings: NIMH Center for the Study of Emotion and Attention, 1999.

G. Zhao, Y. Zhang, Y. Ge, Y. Zheng, X. Sun, and K. Zhang, "Asymmetric hemisphere activation in tenderness: evidence from EEG signals," Scientific Reports, vol. 8, no. 1, pp. 8029-, 2018.

[19] G. Zhao, Y. Zhang, and Y. Ge, "Frontal EEG asymmetry and middle line power difference in discrete emotions," Frontiers in behavioral neuroscience, vol. 12:225. doi: 10.3389/fnbeh.2018.00225, 2018.

[20] Y. J. Liu, M. Yu, G. Zhao, J. Song, G. Yan, and Y. Shi, "RealTime Movie-Induced Discrete Emotion Recognition from EEG Signals," IEEE Transactions on Affective Computing, vol. PP, no. 99, pp. 1-1, 2017.

[21] G. Zhao, Y. Ge, B. Shen, X. Wei, and H. Wang, "Emotion analysis for personality inference from EEG signals," IEEE Transactions on Affective Computing, vol. 9, no. 3, pp. 362371, 2017.

[22] J. J. Gross, and R. W. Levenson, "Emotion elicitation using films," Cognition \& Emotion, vol. 9, no. 1, pp. 87-108, 1995.

[23] M. Soleymani, M. Pantic, and T. Pun, "Multimodal emotion recognition in response to videos," IEEE transactions on affective computing, vol. 3, no. 2, pp. 211-223, 2011.

[24] S. Katsigiannis, and N. Ramzan, "DREAMER: A database for emotion recognition through EEG and ECG signals from wireless low-cost off-the-shelf devices," IEEE journal of biomedical health informatics, vol. 22, no. 1, pp. 98-107, 2017.

[25] W.-L. Zheng, and B.-L. Lu, "Investigating critical frequency bands and channels for EEG-based emotion recognition with deep neural networks," IEEE Transactions on Autonomous Mental Development, vol. 7, no. 3, pp. 162175, 2015.

[26] J. A. M. Correa, M. K. Abadi, N. Sebe, and I. Patras, 
"Amigos: A dataset for affect, personality and mood research on individuals and groups," IEEE Transactions on Affective Computing, 2018.

[27] C. E. Erdem, C. Turan, and Z. Aydin, "BAUM-2: a multilingual audio-visual affective face database," Multimedia Tools Applications, vol. 74 , no. 18, pp. 1-31, 2015.

[28] M. Chen, J. Han, L. Guo, J. Wang, and I. Patras, "Identifying valence and arousal levels via connectivity between EEG channels." pp. 63-69.

[29] X. W. Wang, D. Nie, and B. L. Lu, "EEG-Based Emotion Recognition Using Frequency Domain Features and Support Vector Machines," Neural Information Processing, Pt I, Lecture Notes in Computer Science B. L. Lu, L. Q. Zhang and J. Kwok, eds., pp. 734-+, Berlin: Springer-Verlag Berlin, 2011.

[30] M. Li, Q. Chai, T. Kaixiang, A. Wahab, and H. Abut, "EEG Emotion Recognition System," In-Vehicle Corpus and Signal Processing for Driver Behavior, K. Takeda, J. H. L. Hansen, H. Erdogan and H. Abut, eds., pp. 125-135, Boston, MA: Springer, 2009.

[31] X. Hu, J. W. Yu, M. D. Song, C. Yu, F. Wang, P. Sun, D. F. Wang, and D. Zhang, "EEG Correlates of Ten Positive Emotions," Frontiers in Human Neuroscience, vol. 11, pp. 12, Jan, 2017.

[32] X. Hu, C. Zhuang, F. Wang, Y. J. Liu, C. H. Im, and D. Zhang, "fNIRS Evidence for Recognizably Different Positive Emotions," Frontiers in Human Neuroscience, vol. 13, pp. 11, Apr, 2019.

[33] V. Griskevicius, M. N. Shiota, and S. M. Nowlis, "The Many Shades of Rose-Colored Glasses: An Evolutionary Approach to the Influence of Different Positive Emotions," Journal of Consumer Research, vol. 37, no. 2, pp. 238-250, 2010.

[34] M. N. Shiota, S. L. Neufeld, W. H. Yeung, S. E. Moser, and E. F. Perea, "Feeling Good: Autonomic Nervous System Responding in Five Positive Emotions," Emotion, vol. 11, no. 6, pp. 1368-1378, Dec, 2011.

[35] Y. Deng, M. Yang, and R. Zhou, "A New Standardized Emotional Film Database for Asian Culture," Frontiers in Psychology, vol. 8, pp. 1941, 2017.

[36] R. A. Emmons, and M. E. Mccullough, "Counting blessings versus burdens: an experimental investigation of gratitude and subjective well-being in daily life," Journal of Personality \& Social Psychology, vol. 84, no. 2, pp. 377-89, 2003.

[37] Y. Ge, G. Zhao, Y. Zhang, R. J. Houston, and J. Song, "A standardised database of Chinese emotional film clips," Cognition \& Emotion, vol. 33, no. 5, pp. 976-990, Aug, 2019

[38] P. J. Silvia, "What is interesting? Exploring the appraisal structure of interest," Emotion, vol. 5, no. 1, pp. 89-102, 2005.

[39] R. S. Lazarus, "Hope: An Emotion and a Vital Coping Resource Against Despair," Social Research, vol. 66, no. 2, pp. 653-678, 1999.

[40] O. Christopher, E. J. Horberg, and K. Dacher, "Compassion, pride, and social intuitions of self-other similarity," Journal of Personality and Social Psychology, vol. 98, no. 4, pp. 618-
630, 2010.

[41] A. P. Mcgraw, and C. Warren, "Benign violations: making immoral behavior funny," Psychological Science, vol. 21, no. 8, pp. 1141-1149, 2010.

[42] A. Mossman, "The nature of awe: Elicitors, appraisals, and effects on self-concept," Cognition \& Emotion, vol. 21, no. 5, pp. 944-963, 2007.

[43] D. Keltner, and J. Haidt, "Approaching awe, a moral, spiritual, and aesthetic emotion," Cognition \& Emotion, vol. 17, no. 2, pp. 297-314, 2003.

[44] R. Feldman, "Oxytocin and social affiliation in humans," Hormones \& Behavior, vol. 61, no. 3, pp. 380-391, 2012.

[45] E. M. Tong, "Differentiation of 13 positive emotions by appraisals," Cogn Emot, vol. 29, no. 3, pp. 484-503, 2015.

[46] P. A. Linley, H. Dovey, S. Beaumont, J. Wilkinson, and R. Hurling, "Examining the Intensity and Frequency of Experience of Discrete Positive Emotions," Journal of Happiness Studies, vol. 17, no. 2, pp. 875-892, 2015.

[47] I. Schindler, G. Hosoya, W. Menninghaus, U. Beermann, V. Wagner, M. Eid, and K. R. Scherer, "Measuring aesthetic emotions: A review of the literature and a new assessment tool," Plos One, vol. 12, no. 6, pp. e0178899, 2017.

[48] L. W. Niezink, F. W. Siero, P. Dijkstra, and D. P. H. Barelds, "Empathic concern: Distinguishing between tenderness and sympathy," Motivation \& Emotion, vol. 36, no. 4, pp. 544549, 2012.

[49] E. L. Stocks, D. A. Lishner, and S. K. Decker, "Altruism or psychological escape: Why does empathy promote prosocial behavior? †," European Journal of Social Psychology, vol. 39, no. 5, pp. 649-665, 2010.

[50] D. Dunning, "Normative goals and the regulation of social behavior: The case of respect," Motivation \& Emotion, vol. 41, no. 2, pp. 1-9, 2017.

[51] I. Schindler, "Relations of admiration and adoration with other emotions and well-being," Psychology of Well-Being, vol. 4, no. 1, pp. 1-23, 2014.

[52] N. R. Giuliani, "Craving Is an Affective State and Its Regulation Can Be Understood in Terms of the Extended Process Model of Emotion Regulation," Psychological Inquiry, vol. 26, no. 1, pp. 48-53, 2015.

[53] P. E. Jose, "Appraisal Determinants of Emotions: Constructing a More Accurate and Comprehensive Theory," Cognition \& Emotion, vol. 10, no. 3, pp. 241-278, 1996.

[54] S. Lin, J. Xie, M. Yang, Z. Li, Z. Li, L. Dan, X. Xu, and X. Yang, "A Review of Emotion Recognition Using Physiological Signals," Sensors, vol. 18, no. 7, pp. 2074, 2018.

[55] W. G. Han, S. H. Yu, J. C. Chien, and J. S. Shieh, "Shortterm analysis of heart rate variability for emotion recognition via a wearable ECG device."

[56] W. H. Wen, G. Y. Liu, N. P. Cheng, J. Wei, P. C. Shangguan, and W. J. Huang, "Emotion Recognition Based on MultiVariant Correlation of Physiological Signals," leee Transactions on Affective Computing, vol. 5, no. 2, pp. 126140, Apr-Jun, 2014.

[57] F. Setiawan, S. A. Khowaja, A. G. Prabono, B. N. Yahya, and S. L. Lee, "A Framework for Real Time Emotion Recognition Based on Human ANS Using Pervasive 
Device."

B. L. Fredrickson, "What Good Are Positive Emotions?," Review of General Psychology, vol. 2, no. 3, pp. 300-319, 1998.

L. Fei, L. Yang, H. Shi, and C. Liu, "Differences in photoplethysmography morphological features and feature time series between two opposite emotions: Happiness and sadness," Artery Research, vol. 18, pp. 7-13, 2017.

P. Das, A. Khasnobish, and D. N. Tibarewala, "Emotion recognition employing ECG and GSR signals as markers of ANS."

S. Whiting, S. Moreland, J. Costello, G. Colopy, and C. McCann, Recognising Cardiac Abnormalities in Wearable Device Photoplethysmography (PPG) with Deep Learning, 2018.

C. W. Darrow, and G. R. Gullickson, The Peripheral Mechanism of the Galvanic Skin Response, 1970.

[63] P. Philippot, "Inducing and assessing differentiated emotion-feeling states in the laboratory," Cognition \& Emotion, vol. 7, no. 2, pp. 171-193, 1993.

[64] Z. Qu, J. Chen, B. Li, J. Tan, D. Zhang, and Y. Zhang, "Measurement of high-school students' trait math anxiety using neurophysiological recordings during math exam," IEEE Access, vol. 8, pp. 57460-57471, 2020.

A. Gautam, N. Simoes-Capela, G. Schiavone, A. Acharyya, W. De Raedt, and C. Van Hoof, "A Data Driven Empirical Iterative Algorithm for GSR Signal Pre-Processing." pp. 1162-1166.
H. Lim, B. Kim, G.-J. Noh, and S. K. Yoo, "A deep neural network-based pain classifier using a photoplethysmography signal," Sensors, vol. 19, no. 2, pp. 384; doi:10.3390/s19020384, 2019.

Y. Benjamini, and Y. Hochberg, Controlling The False Discovery Rate - A Practical And Powerful Approach To Multiple Testing, 1995.

R. O. Duda, P. E. Hart, and D. G. Stork, Pattern classification (Second Edition), John Wiley \& Sons, 2001.

M. Charrad, N. Ghazzali, V. Boiteau, A. Niknafs, and M. M. Charrad, "Package 'nbclust'," Journal of statistical software, vol. 61, pp. 1-36, 2014

T. Wei, V. Simko, M. Levy, Y. Xie, Y. Jin, and J. Zemla, "Package 'corrplot'," Statistician, vol. 56, pp. 316-324, 2017.

J. J. Cabibihan, and S. S. Chauhan, "Physiological Responses to Affective Tele-Touch during Induced Emotional Stimuli," leee Transactions on Affective Computing, vol. 8, no. 1, pp. 108-118, Jan-Mar, 2017.

J. Hofmann, T. Platt, and W. Ruch, "Laughter and Smiling in 16 Positive Emotions," leee Transactions on Affective Computing, vol. 8, no. 4, pp. 495-507, Oct-Dec, 2017.

P. Ekman, "Sixteen positive emotions," Emotion Researcher, vol. 18, no. 2, pp. 6-7, 2003.

Yulin Zhang received the B.S. degree in psychology, from Beijing Normal University, China, 2016, and now is a graduate student in the Institute of Psychology, Chinese Academy of Sciences, China. His research interests are emotion recognition based on EEG and physiological signals.
Guozhen Zhao received the B.S. degree in industrial engineering, from Tianjin University, China, in 2007, and the M.S. and Ph.D. degrees in industrial and systems engineering from the State University of New York, Buffalo, in 2009 and 2011, respectively. He is an Associate Professor in the Institute of Psychology, Chinese Academy of Sciences. His current research interest is the mathematical modeling of human cognition and performance, human computer interaction, emotion recognition, and augmented cognition.

Yan Ge received the B.S. degree from Beijing in psychology, from Normal University, China, in 2003, and the Ph.D. degree from Institute of Psychology, Chinese Academy of Sciences in 2008. She is an Associate Professor at Institute of Psychology, Chinese Academy of Sciences. Her main research interest is human-computer interaction.

Yezhi Shu received her B.Eng. degree from Shandong University, China, in 2019, and now she is a Ph.D student with Department of Computer Science and Technology, Tsinghua University. Her research interests include computer vision and cognitive computation.

Dan Zhang received the B.E. degree in automation in 2005 and a Ph.D. degree in biomedical engineering in 2011, both from Tsinghua University. He is an Associate Professor at Department of Psychology, Tsinghua University. He was a postdoctoral fellow in School of Medicine, Tsinghua University from 2011 to 2013. His research interests include social neuroscience, engineering psychology, and brain-computer interfaces. He is a member of the IEEE.

Yong-Jin Liu received the B. Eng. Degree in mechano-electronic engineering from Tianjin University, China, in 1998, and the Ph.D. degree from the Hong Kong University of Science and Technology, Hong Kong, in 2003. He is an Associate Professor with the Department of Computer Science and Technology, Tsinghua University, China. He is a senior member of IEEE. His research interests include pattern analysis, human-computer interaction and computer graphics.

Xianghong Sun received the B.S. degree in mathematics from Wuhan University in 1989, and the Ph.D. degrees in psychology from the Institute of Psychology, Chinese Academy of Sciences in 2001. She is the vice-director and a senior researcher in the Institute of Psychology, Chinese Academy of Sciences. Her current research interest is the human computer interaction, mental workload, and situation awareness 
